\title{
Association between levels of aquaporin 3 in the placenta and adiponectin in the umbilical cord blood with gestational diabetes mellitus and pregnancy outcome
}

\author{
CHUNQI ZHANG, YANXIA LI, JIA WANG, CHUNMEI LIU and YAN CHEN \\ Department of Obstetrics and Gynecology, North China University of Science and \\ Technology Affiliated Hospital, Tangshan, Hebei 063000, P.R. China
}

Received September 5, 2019; Accepted April 28, 2020

DOI: $10.3892 / \mathrm{mmr} .2020 .11225$

\begin{abstract}
Pregnant women with gestational diabetes mellitus (GDM) may have adverse pregnancy outcomes, even if their blood glucose level is well-controlled. Aquaporin 3 (AQP3) and adiponectin (APN) serve important roles in fetal growth and development. However, the associations of AQP3 and APN with GDM and pregnancy outcome are not known. Therefore, the present study was performed to evaluate the expression of AQP3 in the placenta and APN in the umbilical artery blood, and the association of the two factors with GDM and pregnancy outcome. The patient cohort was divided into two groups: Pregnant women with GDM; and pregnant women with normal glucose tolerance (NGT). The expression levels of AQP3 in the placenta and APN in the umbilical artery blood were detected. Logistic regression was used to analyze the associations of AQP3 and APN with GDM and pregnancy outcome. The expression levels of $\mathrm{AQP} 3$ and $\mathrm{AQP} 3$ mRNA in the placenta of the GDM group were decreased compared with that of the NGT group, and the difference was statistically significant $(\mathrm{P}<0.05)$. The expression of APN in the umbilical artery blood of the GDM group was also decreased compared with that of the NGT group, and the difference was also statistically significant $(\mathrm{P}<0.05)$. Multivariate logistic regression analyses indicated that the AQP3 and APN levels were negatively correlated not only with the risk of developing GDM [AQP3 odds ratio $(\mathrm{OR})=5.00(\mathrm{P}<0.01)$; APN OR=2.98 $(\mathrm{P}=0.01)]$, but also with abnormal pregnancy outcome [(AQP3 $\mathrm{OR}=4.64(\mathrm{P}<0.01) ; \mathrm{APN} \mathrm{OR}=5.41 \quad(\mathrm{P}<0.01)]$. The levels of AQP3 in the placenta and APN in the umbilical cord blood were associated with GDM, and the risk of GDM was increased
\end{abstract}

Correspondence to: Professor Yan Chen, Department of Obstetrics and Gynecology, North China University of Science and Technology Affiliated Hospital, 73 Jianshe Road, Tangshan, Hebei 063000, P.R. China

E-mail: yanchen0806@yahoo.com

Key words: gestational diabetes, aquaporin 3, adiponectin, pregnancy outcomes in pregnant women with decreased AQP3 and APN levels. The AQP3 and APN levels also had an effect on pregnancy outcome. The risk of abnormal pregnancy outcomes, including cesarean section, macrosomia, fetal distress and neonatal asphyxia, was increased in pregnant women with decreased AQP3 and APN levels.

\section{Introduction}

Gestational diabetes mellitus (GDM) refers to glucose intolerance with first onset and diagnosis during pregnancy, and is the most common perinatal complication (1). The global prevalence of GDM is expected to increase annually, particularly in Asia (2), possibly due to the observed increase in maternal age and obesity in this continent (3). GDM usually resolves after childbirth, but it is associated with an increased risk of prenatal, perinatal and postnatal adverse events (4). If blood glucose is poorly controlled, GDM may induce hyperglycemia, which affects both the mother and fetus (4). The short-term adverse consequences of hyperglycemia include infection, pre-eclampsia and hypertension for the mother, and birth trauma due to macrosomia for the fetus (5). GDM also has long-term health effects (6). For the mother, the risk of GDM recurrence is increased by $35-50 \%$ in subsequent pregnancies, and $26-70 \%$ of pregnant women with GDM develop type 2 diabetes mellitus within 10-15 years following delivery (5). For the children of mothers with GDM, the risk of developing obesity and type 2 diabetes mellitus increase throughout their lifespan (7), and those born with macrosomia are at an increased risk of cardiovascular disease and leukemia in the future $(4,8)$. However, even if the control of blood glucose level of pregnant women with GDM is satisfactory, the pregnancy outcome may not significantly improved (9). The specific reasons and underlying mechanism remain elusive.

The mother and fetus are connected by the placenta. The placenta is an appendage of the fetus that has major endocrine and transport functions (10). It serves a key role in the growth and development of the fetus, and can synthesize numerous hormones, cytokines and transporters (11). Aquaporin 3 (AQP3) is a subtype of the AQP family, whose functions include solute transport and signal transduction (12). AQP3 is also expressed in the placenta and may transport water and 
glycerol to the fetal circulation. It may also serve an important role in fetal growth and development, and its expression level may be affected by the maternal environment. Hydramnios is a common complication of pregnancy in women with GDM (13). AQP participates in the regulation of amniotic fluid balance, and the AQP level in the placenta is positively correlated with amniotic fluid volume in pregnant women with abnormal glucose metabolism (14). However, for the majority of pregnant women with GDM with a normal amniotic fluid index (AFI), it is unclear whether the expression of placental AQP3 gene is altered, and whether the AQP3 level is associated with AFI. In addition to abnormal glucose metabolism, pregnant women with GDM usually have dysregulated lipid metabolism (15). The AQP3 protein also transports glycerol, which is involved in lipid metabolism (16). However, there are few reports on whether the expression of AQP3 in the placenta is altered, and whether this is associated with GDM.

Adiponectin (APN) is an adipokine secreted by adipose tissue and has insulin-sensitizing, anti-atherosclerotic and anti-inflammatory properties (17). In some cases, APN can also reduce body weight, which is associated with maintaining body metabolism and energy balance (18). Decreases in APN levels serve an important role in obesity-associated diseases, including insulin resistance/diabetes and cardiovascular diseases (19). APN in the umbilical cord blood also serves a key role in regulating fetal growth, development and fat reserves, and is an important index for predicting the pregnancy outcome (20). For pregnant women with GDM, even if the blood glucose is well controlled, the serum APN level is downregulated, and the insulin resistance is increased compared with that of pregnant women with normal glucose tolerance (NGT) (21). However, there are few reports on how APN changes in the umbilical cord blood.

In the present study, the expression of AQP3 in the placenta was detected by immunohistochemistry, reverse transcription-quantitative PCR (RT-qPCR) and western blotting, and the APN level in the umbilical artery blood was determined by ELISA. The associations of AQP3 in the placenta and APN in the umbilical artery blood with GDM and pregnancy outcome were further discussed. The aim was to provide a reference for perinatal health care.

\section{Materials and methods}

Patients. The present study was conducted at the North China University of Science and Technology Affiliated Hospital, between November 2017 and October 2018, and performed in accordance with the ethical guidelines of the Declaration of Helsinki for experiments involving human subjects. The present study was approved by the ethics committee of the North China University of Science and Technology Affiliated Hospital. Informed consent was obtained from all patients. A total of 60 pregnant women with GDM and 60 pregnant women with NGT were recruited. The inclusion criteria for pregnant women with GDM were as follows: A diagnosis of GDM according to oral glucose tolerance test (22) or by documented clinical diagnosis in the medical records; aged 20-40 years; single pregnancy; simple control of blood glucose through diet and exercise; no recent history of taking drugs affecting blood lipid levels; normal pregnancy screenings, such as Down's screening and four-dimensional color Doppler ultrasound; complete clinical data; and the provision of informed consent. The exclusion criteria were: Unsatisfactory blood glucose control; and patients with pregnancy complications such as thyroid disease, pregnancy-induced hypertension, or severe heart, liver, kidney and other diseases. The criteria for satisfactory blood glucose control during pregnancy were as follows: Blood glucose should be 5.3-6.7 mmol/1 prior and subsequent to meals; blood glucose at night should not be $<3.3 \mathrm{mmol} / \mathrm{l}$; and glycosylated hemoglobin during pregnancy should not be $<5.5 \%$ (23). The inclusion criteria for the patients with NGT were as: Aged 20-40 years; single pregnancy; no recent history of taking drugs affecting blood lipid levels; normal pregnancy screenings, such as Down's screening and four-dimensional color Doppler ultrasound; complete clinical data; and provision of informed consent. The exclusion criteria were: Diagnosis of pre-pregnancy diabetes (including types 1 and 2); control of blood glucose using drugs; and patients with pregnancy complications such as thyroid disease, pregnancy-induced hypertension, and severe heart, liver, kidney and other diseases.

There were four pregnancy outcomes in the present study: i) Macrosomia, defined as fetal weight $\geq 4,000 \mathrm{~g}$; ii) fetal distress, defined as type III electronic fetal heart rate monitoring graphics, meconium contamination of the amniotic fluid, or abnormal fetal movement; iii) neonatal asphyxia, defined as (a) 5 min Apgar score $<7$ points and no effective breathing established; (b) umbilical artery blood gas $\mathrm{pH}<7.15$; (c) exclusion of other causes of low Apgar score; (d) the presence of prenatal high-risk factors that may lead to asphyxia ( $\mathrm{a}, \mathrm{b}$ and $\mathrm{c}$ were the necessary conditions, $\mathrm{d}$ was the reference condition); and iv) cesarean section, defined as an obstetric surgical procedure for abnormal delivery, indicating scarred uterus, cephalopelvic disproportion or fetal distress. Any cases of macrosomia, fetal distress, neonatal asphyxia or cesarean section were considered as abnormal outcomes; otherwise, the outcomes were considered as normal.

\section{Blood, tissue samples and biochemical analyses}

Placental tissue collection. Immediately following delivery, 3 placental tissue samples $\sim 1 \mathrm{~cm}^{3}$ were removed from the central non-calcified area of the placenta; after washing with normal saline, 1 of the samples was fixed with $10 \%$ neutral formalin at room temperature for 1 day for immunohistochemical study, and the remaining 2 samples were placed in a freezing tube and stored in a freezer at $-80^{\circ} \mathrm{C}$ for western blotting and RT-qPCR analyses.

Umbilical artery blood collection. Within 1 min after delivery, $3 \mathrm{ml}$ umbilical artery blood was extracted by syringe and injected into a coagulation-promoting tube; serum was then separated by centrifugation $\left(1,000 \times \mathrm{g}\right.$ at $4^{\circ} \mathrm{C}$ for $\left.15 \mathrm{~min}\right)$, sealed and stored in the refrigerator at $-80^{\circ} \mathrm{C}$ for detection of APN.

Biochemical analyses. Routine blood tests, including pre-delivery fasting plasma glucose (FPG), glycosylated hemoglobin (HbAlc) and serum triglyceride (TG) were completed by the Laboratory Department of North China University of Science and Technology Affiliated Hospital. Clinical 
Table I. Clinical data analysis results of the two groups.

\begin{tabular}{lccc}
\hline Characteristics & GDM $(\mathrm{n}=60)$ & NGT $(\mathrm{n}=60)$ & P-value \\
\hline Age, years & $30.57 \pm 4.83$ & $29.85 \pm 4.07$ & 0.38 \\
BMI prior to pregnancy & $23.23 \pm 4.34$ & $22.22 \pm 3.88$ & 0.18 \\
Gestational week, weeks & $39.04 \pm 0.88$ & $39.11 \pm 0.90$ & 0.64 \\
Number of pregnancies, $\mathrm{n}$ & $2.75 \pm 2.00$ & $2.17 \pm 1.42$ & 0.07 \\
Number of births, $\mathrm{n}$ & $0.63 \pm 0.71$ & $0.43 \pm 0.50$ & 0.08 \\
Gestational weight gain, kg & $17.60 \pm 4.97$ & $14.66 \pm 4.60$ & 0.00 \\
FPG, mmol/1 & $4.50 \pm 0.64$ & $4.35 \pm 0.60$ & 0.18 \\
HbA1c, $\%$ & $5.04 \pm 0.41$ & $4.90 \pm 0.44$ & 0.08 \\
TG, mmol/l & $3.85 \pm 1.64$ & $2.93 \pm 1.15$ & 0.00 \\
AFI, cm & $6.71 \pm 1.67$ & $6.83 \pm 2.04$ & 0.88 \\
Neonatal weight, g & $3,619.17 \pm 384.78$ & $3,434.00 \pm 493.37$ & 0.02 \\
\hline
\end{tabular}

Data are presented as the mean \pm standard deviation. GDM, gestational diabetes mellitus; NGT, normal glucose tolerance; BMI, body mass index; FPG, fasting plasma glucose; HbA1c, glycosylated hemoglobin; TG, serum triglyceride; AFI, amniotic fluid index.
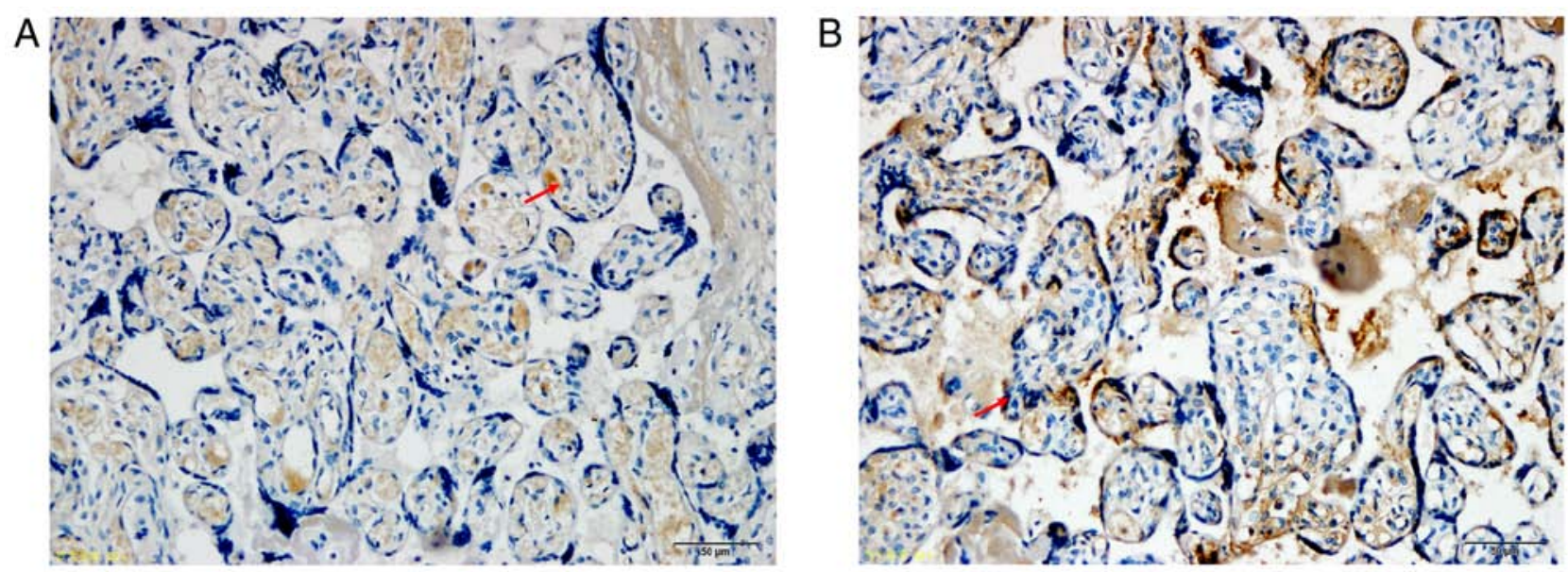

Figure 1. Immunohistochemical examination for AQP3 in the (A) GDM and (B) NGT groups. Samples were considered to be AQP3-positive when brown-yellow granules were detected in the cytoplasm and cell membrane (red arrows). Magnification, $\mathrm{x} 400$. Scale bar=50 $\mu \mathrm{m}$. AQP3, aquaporin 3; GDM, gestational diabetes mellitus; NGT, normal glucose tolerance.

examination data, including AFI and neonatal weight, were obtained in the Department of Obstetrics and Gynecology. The APN level in the umbilical artery serum was detected using commercial ELISA kits (cat. no. 20180906451; Shanghai YL Biotech Co., Ltd.) according to the manufacturer's protocol.

Immunohistochemistry analysis. Formalin-fixed (at $4^{\circ} \mathrm{C}$ for $24 \mathrm{~h}$ ) placental tissue samples were dehydrated through graded alcohols (50, 60, 80, 90, 95 and 100\% alcohol), embedded in paraffin, and cut into sections $(3 \times 3 \times 3 \mathrm{~mm})$. After routinely dewaxing and hydration $(100,95,85$ and $75 \%$ alcohol), antigen retrieval was performed in EDTA antigen retrieval solution (Shanghai Guangrui Biotechnology Co., Ltd.; http://www.shgrsw.com/.). Subsequently, endogenous peroxidase activity was blocked by incubating the slides with $3 \%$ $\mathrm{H}_{2} \mathrm{O}_{2}$ for $15 \mathrm{~min}$ at room temperature. The sections were incubated with rabbit anti-human AQP3 antibody (cat. no. AF5222, Affinity BioReagents, Inc.; 1:200) at $4^{\circ} \mathrm{C}$ overnight and $37^{\circ} \mathrm{C}$ for $40 \mathrm{~min}$, and followed by incubation with goat anti-rabbit IgG horseradish peroxidase (HRP)-conjugated secondary antibodies (cat. no. ZB-2301; 1:20; OriGene Technologies, Inc.) at $37^{\circ} \mathrm{C}$ for $30 \mathrm{~min}$. The color developing was performed with DAB color developer solution (OriGene Technologies, Inc.) and the sections were stained with hematoxylin at room temperature for $30 \mathrm{sec}$. Immunohistochemical staining images (magnification, x400) were obtained by a Micro Publisher 5.0 microscope (Roper Industries). Image-Pro-Plus 6.0 software (Media Cybernetics, Inc.) was used to analyze the results of immunohistochemistry and calculate the integral optical density. A positive result for the AQP3 protein staining was the presence of brown granules in the cell membrane and cytoplasm.

Western blotting. Total cytoplasmic protein was extracted from placental tissue using RIPA lysis buffer (BestBio Co., Ltd.; http://www.bestbio.com.cn/) and quantified using a 
Table II. Comparison of pregnancy outcomes between the two groups

n

\begin{tabular}{lccccc}
\cline { 2 - 5 } Outcome & Total & GDM & NGT & $\chi^{2}$ & P-value \\
\hline Cesarean section & 63 & 34 & 29 & 0.84 & 0.36 \\
Macrosomia & 15 & 12 & 3 & 4.88 & 0.03 \\
Fetal distress & 7 & 4 & 3 & 0.00 & 1.00 \\
Neonatal asphyxia & 3 & 2 & 1 & 0.00 & 1.00 \\
\hline
\end{tabular}

GDM, gestational diabetes mellitus; NGT, normal glucose tolerance.

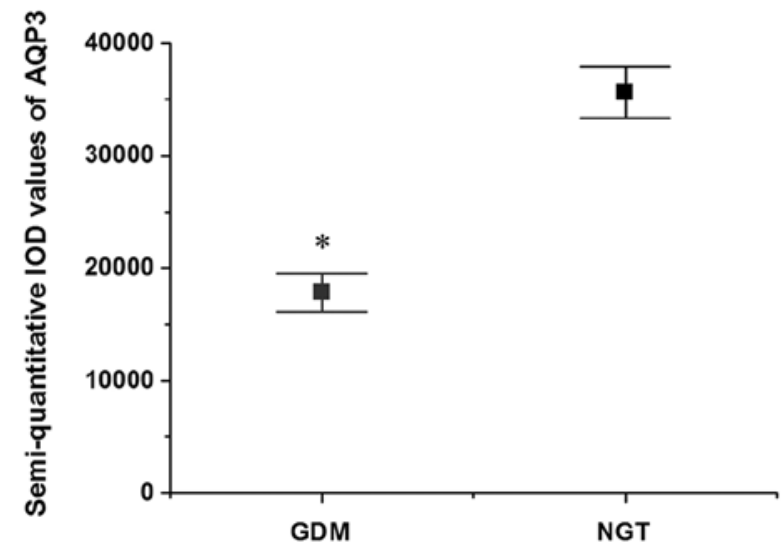

Figure 2. Immunohistochemical semi-quantitative IOD values of placental tissues in the two groups. ${ }^{*} \mathrm{P}<0.05$ vs. NGT. IOD, integrated optical density; AQP3, aquaporin 3; GDM, gestational diabetes mellitus; NGT, normal glucose tolerance.

BCA Protein Assay kit (Shanghai Guangrui Biotechnology Co., Ltd.). Protein (20 $\mu \mathrm{l} /$ lane) was denatured and resolved by $8 \%$ SDS-PAGE (Shanghai Huyu Biotechnology Co., Ltd.; http://www.shhymall.com/.), and transferred at $240 \mathrm{~mA}$ for $2 \mathrm{~h}$ onto a PVDF membrane (Bio-Rad Laboratories, Inc.). Non-specific regions of the PVDF membrane were blocked by incubating the membrane with $5 \%$ skimmed milk at room temperature for $2 \mathrm{~h}$. Then, the PVDF membrane was probed with primary rabbit anti-human AQP3 antibody (cat. no. sc518001; Santa Cruz Biotechnology Inc.; 1:1,000) or rabbit anti-human $\beta$-actin antibody (cat. no. sc58675; Santa Cruz Biotechnology Inc.; 1:5,000) overnight at $4^{\circ} \mathrm{C}$, followed by incubation with goat anti-rabbit IgG HRP-conjugated secondary antibodies (cat. no. 111-035-003; Jackson ImmunoResearch Laboratories, Inc.; 1:2,000) for $2 \mathrm{~h}$ at room temperature. Finally, detection of protein signals was performed using an ECL Chemiluminescence kit (GrBio), and observed by Image Lab5.0 software (Bio-Rad Laboratories, Inc.). To obtain the relative expression of AQP3, the AQP3 signal was normalized to the $\beta$-actin signal.

$R T$ - $q P C R$. Total RNA was extracted form placental tissue using TRIzol ${ }^{\circledR}$ reagent (Invitrogen; Thermo Fisher Scientific, Inc.). RNA was reversely transcribed to cDNA by the M5 First Strand cDNA Synthesis kit (Mei5bio; Beijing

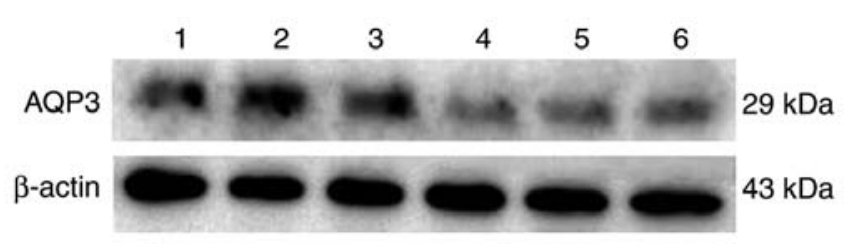

Figure 3. Expression of AQP3 in the gestational diabetes mellitus (lanes 1, 2 and 3 ) and the normal glucose tolerance groups (lanes 4, 5 and 6). AQP3, aquaporin 3 .

Jumei Biotechnology Co., Ltd.) according to the manufacturer's protocol. Then, qPCR was performed by the SYBR Green Realtime PCR Mix kit (Mei5bio; Beijing Jumei Biotechnology Co., Ltd.). $\beta$-actin was selected as an internal reference. The following primers were used: AQP3 forward: 5'-GAAGTC AGGTCATAAGTT-3' and reverse: 5'-GAAGTC AGGTCATAAGTT-3'; $\beta$-actin forward: 5'-GAAGTCAGG TCATAAGTT-3' and reverse: 5'-ACTCTTCCAGCCTTC CTT-3'. The PCR conditions were as follows: Denaturation of the DNA at $95^{\circ} \mathrm{C}$ for $10 \mathrm{~min}$, followed by denaturing at $95^{\circ} \mathrm{C}$ for $15 \mathrm{sec}$, annealing at $65^{\circ} \mathrm{C}$ for $15 \mathrm{sec}$, and extension at $72^{\circ} \mathrm{C}$ for $60 \mathrm{sec}$ for 40 cycles. The value of $2^{-\Delta \Delta \mathrm{Cq}}(\Delta \mathrm{Cq}=\mathrm{Cq}$ value of $\mathrm{APQ} 3$ - $\mathrm{Cq}$ value of $\beta$-actin; $\Delta \Delta \mathrm{Cq}=\mathrm{Cq}$ value of GDM group - Cq value of NGT group) was used as the relative expression of AQP3 mRNA and calculated according to a previous report (24).

Statistical analysis. Statistical analyses were performed using SPSS 23.0 statistical software (IBM Corp.). A Mann-Whitney $\mathrm{U}$ test was used to analyze the immunohistochemistry results. Quantitative data are presented as mean \pm standard deviation, and the results between two groups were compared with Student's t-test. Classified count data were analyzed using the $\chi^{2}$ test. The associations of AQP3 and APN with GDM and pregnancy outcome were analyzed by logistic regression analysis. $\mathrm{P}<0.05$ was considered to indicate a statistically significant difference.

\section{Results}

Clinical data analysis. As demonstrated in Table I, there were no significant differences in age, pre-pregnancy body mass index, gestational week, number of pregnancies, number of 
Table III. Univariate logistic regression analysis of factors affecting the development of GDM in patients.

\begin{tabular}{|c|c|c|c|c|c|c|c|}
\hline \multicolumn{8}{|l|}{ A, AQP3 } \\
\hline Patient group & $\mathrm{n}$ & High level $\left(\geq 0.78^{a}\right)$ & Low level $(<0.78)$ & $\chi^{2}$ & P-value & OR & $95 \% \mathrm{CI}$ \\
\hline GDM & 60 & 14 & 46 & 18.04 & 0.00 & 5.29 & $2.39-11.68$ \\
\hline NGT & 60 & 37 & 23 & & & & \\
\hline
\end{tabular}

$\mathrm{B}, \mathrm{APN}, \mathrm{mg} / \mathrm{l}$

\begin{tabular}{|c|c|c|c|c|c|c|c|}
\hline Patient group & $\mathrm{n}$ & High level ( $\geq 49.93)$ & Low level $(<49.93)$ & $\chi^{2}$ & P-value & OR & $95 \% \mathrm{CI}$ \\
\hline GDM & 60 & 22 & 38 & 8.53 & 0.00 & 2.98 & $1.42-6.27$ \\
\hline NGT & 60 & 38 & 22 & & & & \\
\hline
\end{tabular}

${ }^{a}$ Mean relative expression levels of AQP3 (0.78) from western blotting results. AQP3, aquaporin 3; APN, adiponectin; GDM, gestational diabetes mellitus; NGT, normal glucose tolerance; OR, odds ratio; CI, confidence interval.

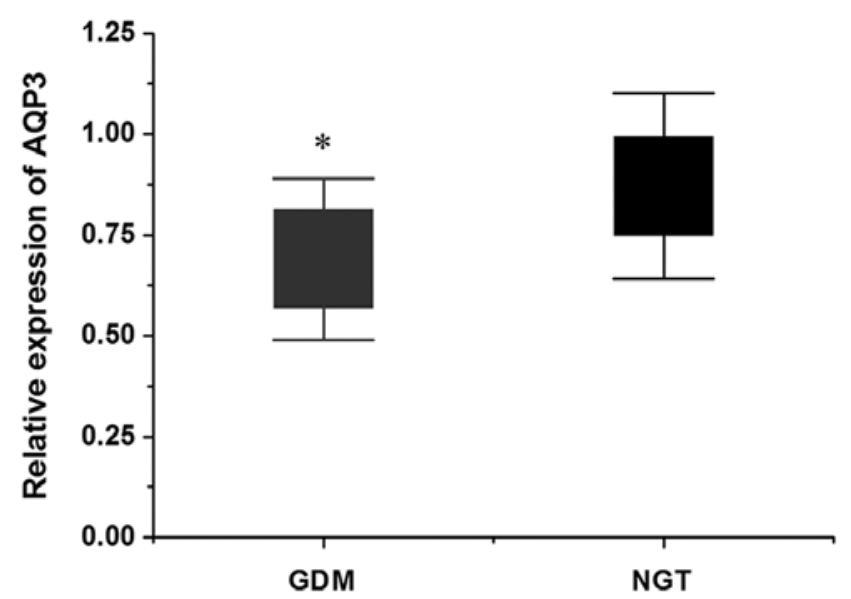

Figure 4. Relative protein expression of AQP3 in placental tissues of the two groups. ${ }^{*} \mathrm{P}<0.05$ vs. NGT. AQP3, aquaporin 3; GDM, gestational diabetes mellitus; NGT, normal glucose tolerance.

births, pre-delivery FPG, HbAlc and AFI between the GDM and NGT groups $(\mathrm{P}>0.05)$. The gestational weight gain, TG and neonatal weight in the GDM group were increased compared with those in the NGT group, and the differences were statistically significant $(\mathrm{P}<0.05)$.

As shown in Table II, the pregnancy outcomes of cesarean section, macrosomia, fetal distress and neonatal asphyxia were compared between the GDM and NGT groups. The incidence of macrosomia in the GDM group was significantly increased compared with that in the NGT group $(\mathrm{P}<0.05)$, but there was no significant difference in the incidence of cesarean section, fetal distress and neonatal asphyxia. This demonstrated that good control of blood glucose levels in pregnant women with GDM may not singularly improve pregnancy outcomes.

$A Q P 3$ and APN levels. As shown in Fig. 1, the AQP3 protein, which was expressed in the cytoplasm and membrane of placental trophoblasts, was detected as brown and yellow granules, and its expression was weakly positive in the GDM group and positive in the NGT group. According to

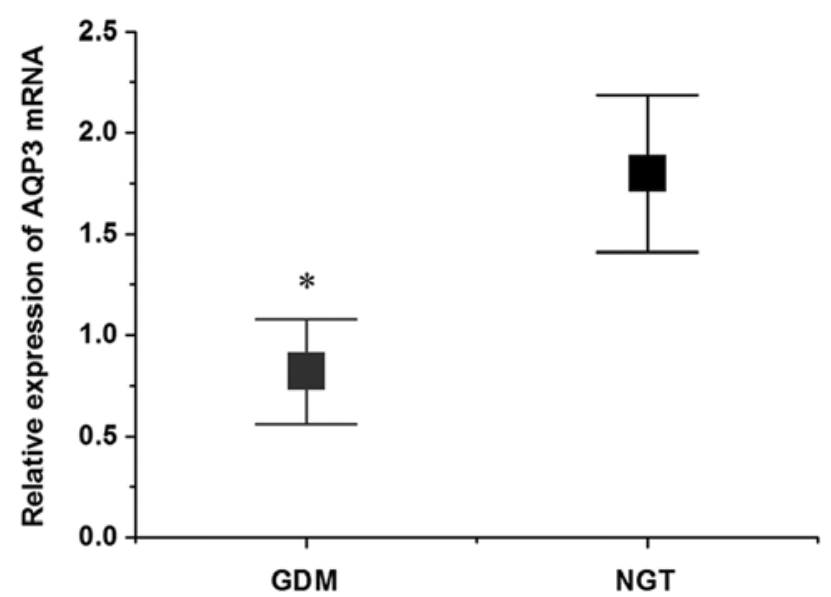

Figure 5. Relative expression of AQP3 mRNA in placental tissues of the two groups. "P<0.05 vs. NGT. AQP3, aquaporin 3; GDM, gestational diabetes mellitus; NGT, normal glucose tolerance.

the semi-quantitative results (Fig. 2), the difference was statistically significant $(\mathrm{P}<0.05)$. According to the western blotting results (Fig. 3), the expression of the AQP3 protein in placental tissues of the GDM group was also significantly decreased compared with that in the NGT group (Fig. 4). The RT-qPCR results demonstrated that the relative expression of AQP3 mRNA in placental tissues of the GDM group was decreased compared with that of the NGT group, and the difference was statistically significant ( $\mathrm{P}<0.05$; Fig. 5). The results of immunohistochemistry, western blotting and RT-qPCR were consistent. The APN level in the umbilical artery serum was detected by ELISA. As shown in Fig. 6, the APN level in the GDM group was decreased compared with that in the NGT group, and the difference was statistically significant $(\mathrm{P}<0.05)$.

Association analysis. As shown in Table III, the mean relative expression levels of AQP3 in the placenta (0.78) and the mean APN level in the umbilical artery serum $(49.93 \mathrm{mg} / \mathrm{l})$ were used as the threshold between high and low levels. Univariate 
Table IV. Multivariate logistic regression analysis of factors affecting the development of GDM in patients.

\begin{tabular}{lccrrrr}
\hline Factor & B & SE & Wals $\chi^{2}$ & P-value & OR & $95 \%$ CI \\
\hline AQP3 & 1.61 & 0.44 & 13.64 & $<0.01$ & 5.00 & $2.13-11.75$ \\
APN & 1.09 & 0.43 & 6.51 & 0.01 & 2.98 & $1.29-6.89$ \\
TG & 1.25 & 0.43 & 8.41 & $<0.01$ & 3.48 & $1.50-8.10$ \\
\hline
\end{tabular}

AQP3, aquaporin 3; APN, adiponectin; TG, serum triglyceride; OR, odds ratio; CI, confidence interval.

Table V. Univariate logistic regression analysis of factors affecting pregnancy outcome.

\begin{tabular}{|c|c|c|c|c|c|c|c|c|}
\hline \multicolumn{9}{|l|}{ A, AQP3 } \\
\hline Patient group & Pregnancy outcome & $\mathrm{n}$ & High level $\left(\geq 0.78^{a}\right)$ & Low level $(<0.78)$ & $\chi^{2}$ & P-value & OR & $95 \% \mathrm{CI}$ \\
\hline \multirow[t]{2}{*}{ GDM } & Normal & 24 & 2 & 22 & 3.73 & 0.04 & 5.50 & $1.11-27.37$ \\
\hline & Abnormal & 36 & 12 & 24 & & & & \\
\hline \multirow[t]{2}{*}{ NGT } & Normal & 20 & 5 & 15 & 17.06 & $<0.01$ & 12.00 & $3.35-42.93$ \\
\hline & Abnormal & 40 & 32 & 8 & & & & \\
\hline
\end{tabular}

$\mathrm{B}, \mathrm{APN}, \mathrm{mg} / \mathrm{l}$

\begin{tabular}{|c|c|c|c|c|c|c|c|c|}
\hline Patient group & Pregnancy outcome & $\mathrm{n}$ & High level $(\geq 49.93)$ & Low level $(<49.93)$ & $\chi^{2}$ & $\mathrm{P}$-value & OR & $95 \% \mathrm{CI}$ \\
\hline \multirow[t]{2}{*}{ GDM } & Normal & 24 & 5 & 19 & 4.32 & 0.04 & 3.40 & $1.04-11.09$ \\
\hline & Abnormal & 36 & 17 & 19 & & & & \\
\hline \multirow[t]{2}{*}{ NGT } & Normal & 20 & 4 & 16 & 24.26 & 0.00 & 22.67 & $5.60-91.71$ \\
\hline & Abnormal & 40 & 34 & 6 & & & & \\
\hline
\end{tabular}

${ }^{\mathrm{a}}$ Mean relative expression levels of AQP3 (0.78) from western blotting results. AQP3, aquaporin 3; APN, adiponectin; GDM, gestational diabetes mellitus; NGT, normal glucose tolerance; OR, odds ratio; CI, confidence interval.

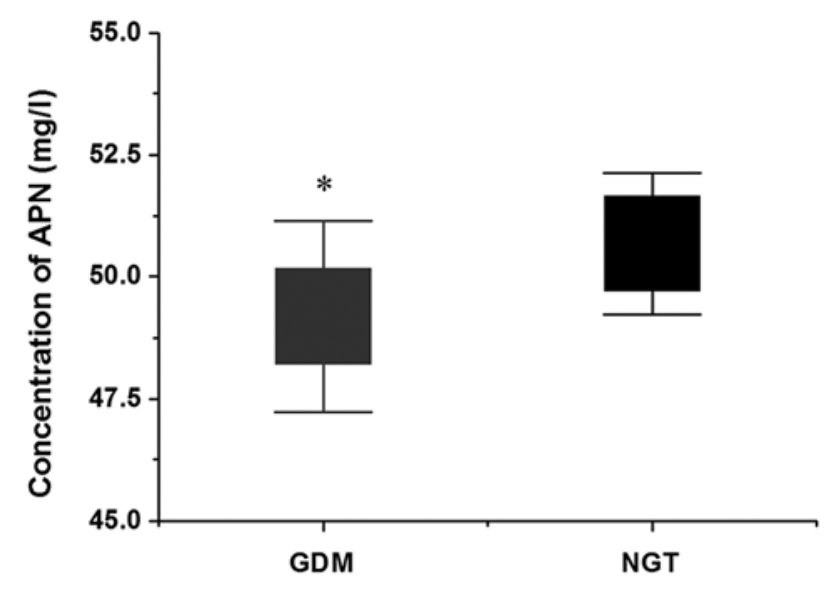

Figure 6. Concentration of APN in the umbilical arterial blood of the two groups. ${ }^{*} \mathrm{P}<0.05$ vs. NGT. APN, adiponectin; GDM, gestational diabetes mellitus; NGT, normal glucose tolerance.

logistic regression analysis revealed that the expression levels of AQP3 and APN were associated with GDM. The risk of GDM in pregnant women with low expression levels of AQP3 and APN were increased 5.29- and 2.98-fold compared with those in the women with high levels of AQP3 and APN, respectively.

To investigate the risk factors of GDM, the indicators that differed significantly between the GDM and NGT groups, including AQP3, APN, TG, gestational weight gain and neonatal weight, were selected as independent variables, and their means were used as the threshold between high and low levels. As shown in Table IV, multivariate logistic regression analysis indicated that AQP3, APN and TG were statistically significant different $(\mathrm{P}<0.05)$, and the odds ratios were $5.00,2.98$ and 3.48, respectively. Their corresponding $95 \%$ confidence intervals were $2.13-11.75,1.29-6.89$ and 1.50-8.10, respectively.

As shown in Table $\mathrm{V}$, univariate logistic regression analysis demonstrated that the AQP3 level was significantly associated with pregnancy outcome; the risk of abnormal pregnancy outcome in the GDM and NGT groups with low levels of AQP3 expression was increased 5.50- and 12.00-fold, respectively, compared with that in the respective groups with high AQP3 expression. Similarly, the APN level also had a significant effect on pregnancy outcomes; the risk of abnormal pregnancy outcome in the GDM and NGT groups with low levels of APN was increased 3.40- and 22.67-fold, respectively, 
Table VI. Multivariate logistic regression analysis of factors affecting pregnancy outcome.

\begin{tabular}{lccrcrr}
\hline Factor & $\mathrm{B}$ & $\mathrm{SE}$ & Wals $\chi^{2}$ & $\mathrm{P}$ & OR & $95 \%$ CI \\
\hline AQP3 & 1.53 & 0.51 & 9.24 & 0.00 & 4.64 & $1.72-12.46$ \\
APN & 1.69 & 0.47 & 12.87 & 0.00 & 5.41 & $2.15-13.61$ \\
\hline
\end{tabular}

AQP3, aquaporin 3; APN, adiponectin; OR, odds ratio; CI, confidence interval.

compared with that in the respective groups with high APN expression. The AQP3 and APN levels in the NGT group were more closely associated with pregnancy outcome compared with those in the GDM group, possibly due to other factors affecting pregnancy outcome in the GDM group.

Multivariate logistic regression analysis was conducted with abnormal pregnancy outcome as the dependent variable and AQP3 and APN as independent variables. As shown in Table VI, the results indicated that both the AQP3 and APN levels significantly affected pregnancy outcome. The risk of abnormal pregnancy outcome in pregnant women with low levels of AQP3 and APN expression was increased 4.64- and 5.41-fold, respectively, compared with that associated with high levels of AQP3 and APN expression.

\section{Discussion}

GDM may be caused by a number of factors. For example, in normal pregnancy, levels of insulin resistance may approach those normally observed in patients with type 2 diabetes mellitus and can be inhibited by excessive secretion of $\beta$ cells. However, if $\beta$ cells are unable to secrete enough insulin, pregnant women are more likely to develop GDM (25). The pathogenesis of GDM also includes genetic factors, inflammatory factors, adipokines and decreased expression of estrogen receptors $(26,27)$. Changes in lipid metabolism are a predictor of GDM. Excessive free fatty acids can decrease the sensitivity of surrounding tissues to insulin, cause insulin resistance, and subsequently lead to GDM (28). Therefore, the blood lipid levels of pregnant women with GDM are generally increased. As maternal serum TG and free fatty acids are associated with fetal blood lipids, fetal growth and fat quality, fatty acids and glycerol may affect fetal growth and fat quality, which may lead to abnormal pregnancy outcomes, including cesarean section, macrosomia, fetal distress and neonatal asphyxia $(29,30)$. In the present study, there was no significant difference in FPG and HbA1c between the GDM and NGT groups, suggesting that the blood glucose in the GDM group was well-controlled. However, the blood TG level of the GDM group was significantly increased compared with that of the NGT group, and gestational weight gain and neonatal weight values were both increased compared with those in the GDM group. Therefore, although the blood glucose control was satisfactory, the levels of insulin resistance and lipid metabolism of the pregnant women with GDM did not improve, and the blood TG level in the GDM group remained increased compared with the NTG group. This result suggested that pregnant women with GDM cannot alter their rates of lipid metabolism or weight gain by controlling blood glucose alone. In addition, the incidence of macrosomia in the GDM group was significantly increased compared with that in the NGT group. This indicated that the pregnancy outcome of GDM was not only associated with maternal blood glucose, but also with other factors, such as blood TG. Although the incidence of macrosomia increased in the GDM group, there was no significant difference in the cesarean section rate between the two groups, which may be associated with the larger proportion of uterine scarring in the GDM group. The incidence of fetal distress and neonatal asphyxia was not statistically significantly different, which may be attributed to the small sample.

$\mathrm{AQP}$ is an integral membrane protein family that includes 13 subtypes (12). The AQP8 and AQP8 mRNA levels in GDM are associated with amniotic fluid volume, and increase concomitantly with the increase in the amniotic fluid volume $(31,32)$. In the present study, there was no significant difference in the AFI values between the NGT and GDM groups with good blood glucose control. However, the expression level of the AQP3 protein in the placenta of the GDM group was significantly decreased compared with that of the NGT group, indicating that the level of AQP in the placenta of the GDM group with normal AFI and good blood sugar control may still be affected. Therefore, the placental AQP level is not associated with AFI, and blood glucose level exerts no significant effect on AQP level.

Microarray global gene expression analysis revealed that the GDM placenta has 66 differentially expressed genes, involving not only AQPs, but also cell activation, immune response, organ development and regulation of cell death (33). In the present study, the blood TG level of the GDM group was significantly increased compared with that of the NGT group, and the expression of AQP3 in the placenta of the GDM group was significantly decreased compared with that in the NGT group. The fetuses of pregnant women with GDM exhibited macrosomia, with a high transfer rate of water and glycerol in the placenta. As a negative feedback of fetal overgrowth, the AQP3 level in the GDM placenta was downregulated, which may decrease the transport of maternal glycerol to the fetus through the placenta. Accordingly, the reduction of glycerol transported to the fetus increased the level of free glycerol in the maternal circulation, which, in turn, increased the level of TG in the blood of pregnant women with GDM. In addition, downregulation of AQP3 may result in impaired intestinal barrier integrity by opening tight junction complexes, suggesting that the effect of AQP3 may be more complex than the simple facilitation of membrane permeability (34). Therefore, it may be inferred that AQP3 serves an important role in glycerol diffusion and placental lipid metabolism in GDM, and a regulatory role in fetal growth and development. 
APN, as an adipokine that regulates sugar and lipid metabolism, is closely associated with GDM (35). As fetal growth and development are affected by insulin and glucose metabolism, APN is also an important factor in regulating fetal intrauterine development (36), and the APN level in the umbilical cord blood was identified to be negatively correlated with neonatal weight (37). In the present study, the APN level in the umbilical cord blood of the GDM group was significantly decreased compared with that of the NGT group, demonstrating that pregnant women with lower levels of APN in the umbilical cord blood are at an increased risk of developing GDM. The neonatal weight in the GDM group was significantly increased compared with that in the NGT group, indicating that the decrease in APN in the umbilical cord blood may be associated with the increase of the fetal fat reserve in the uterus.

During pregnancy, APN can recognize its receptor, induce protein kinase activation, increase the production of intracellular cAMP through the protein kinase A pathway, and then induce trophoblast differentiation through cAMP (38). As the transport, gating and redistribution of AQP3 are regulated by phosphorylation, and the phosphorylation of AQP3 is dependent on cAMP (39), an increase in intracellular cAMP may promote the phosphorylation of AQP3, thereby increasing the transport of water and glycerol through the cell membrane. Therefore, APN in the maternal serum may act on placental trophoblasts and increase the activity of AQP3 in placental tissues through the cAMP-PKA pathway. In addition, APN receptor signaling is transmitted by peroxisome proliferator activated receptor (PPAR), and AQP3 is the target of PPAR (40). In the present study, the downregulation of AQP3 expression in the GDM group decreased the regulation of APN on glucose and fat metabolism. The levels of AQP3 in the placenta and of APN in the umbilical artery blood were identified to be negatively association with GDM and pregnancy outcomes: The decrease in AQP3 and APN levels increased the risks of developing GDM and abnormal pregnancy outcomes. Unfortunately, the evaluation model could not be established in the present study, as there were numerous factors affecting pregnancy outcome, blood lipids values and other indicators, and there were only a few indicators examined. Thus, the authors plan to establish an evaluation model and prediction model that could evaluate the outcome of pregnancy in future studies.

In conclusion, the level of AQP3 expression in the placenta was identified to be associated with GDM. The decrease in AQP3 expression levels in the placenta increased the risk of developing GDM. It was inferred that the expression of AQP3 in the placenta was associated with lipid metabolism at the placental interface in patients with GDM. The specific mechanism of AQP3 regulation of fetal growth and development requires further investigation. The decrease in the APN level in the umbilical artery blood was also identified to be associated with GDM and was an important factor in regulating fetal intrauterine development. In addition, low levels of AQP3 in the placenta and APN in the umbilical cord blood increased the risk of abnormal pregnancy outcomes, such as cesarean section, macrosomia, fetal distress and neonatal asphyxia. Understanding these risk factors of GDM may be helpful to identify high-risk pregnant women, formulate effective preventive measures, and provide a basis for the effective management of GDM. The limitations of the present study included a lack of analysis of the maternal pregnancy outcome and establishment of an evaluation model, which will be included in future studies.

\section{Acknowledgements}

Not applicable.

\section{Funding}

No funding was received.

\section{Availability of data and materials}

The datasets used and/or analyzed during the current study are available from the corresponding author on reasonable request.

\section{Authors' contributions}

CZ and YC designed the study. CZ, YL and JW performed the experiments. CL analyzed the data. $\mathrm{CZ}$ and $\mathrm{YC}$ wrote the manuscript. All authors read and approved the final manuscript.

\section{Ethics approval and consent to participate}

The present study was approved by the ethics committee of the North China University of Science and Technology Affiliated Hospital. Informed consent was obtained from all patients.

\section{Patient consent for publication}

All the individuals who participated in the study provided written informed consent for the publication of any associated data.

\section{Competing interests}

The authors declare that they have no competing interests.

\section{References}

1. Gilbert L, Gross J, Lanzi S, Quansah DY, Puder J and Horsch A: How diet, physical activity and psychosocial well-being interact in women with gestational diabetes mellitus: An integrative review. BMC Pregnancy Childbirth 19: 60, 2019.

2. Lee KW, Ching SM, Ramachandran V, Yee A, Hoo FK, Chia YC, Wan Sulaiman WA, Suppiah S, Mohamed MH and Veettil SK: Prevalence and risk factors of gestational diabetes mellitus in Asia: A systematic review and meta-analysis. BMC Pregnancy Childbirth 18: 494, 2018.

3. Laine MK, Kautiainen H, Gissler M, Raina M, Aahos I, Järvinen K, Pennanen P and Eriksson JG: Gestational diabetes in primiparous women-impact of age and adiposity: A register-based cohort study. Acta Obstet Gynecol Scand 97: 187-194, 2018

4. Harrison AL, Shields N, Taylor NF and Frawley HC: Exercise improves glycaemic control in women diagnosed with gestational diabetes mellitus: A systematic review. J Physiother 62: 188-196, 2016.

5. McIntyre HD, Catalano P, Zhang C, Desoye G, Mathiesen ER and Damm P: Gestational diabetes mellitus. Nat Rev Dis Primers 5: 47, 2019.

6. Zamanfar D, Farhadi R, Shahbaznejad L: Neonate of diabetic mother, pathogenesis and complications. J Clin Excell 2, 90-103, 2014 (In Persian). 
7. Scholtens DM, Kuang A, Lowe LP, Hamilton J, Linder B, Lawrence JM, Lebenthal Y, McCance D, Nodzenski M, Talbot $\mathrm{O}$, et al: Hyperglycemia and adverse pregnancy outcome follow-up study (hapo fus): Maternal gestational diabetes mellitus and childhood glucose metabolism. Diabetes Care 42: 381-392, 2019.

8. Walsh JM and McAuliffe FM: Prediction and prevention of the macrosomic fetus. Eur J Obstet Gynecol Reprod Biol 162 : 125-130, 2012.

9. Fantinelli S, Marchetti D, Verrocchio MC, Franzago M, Fulcheri $\mathrm{M}$ and Vitacolonna E: Assessment of psychological dimensions in telemedicine care for gestational diabetes mellitus: A systematic review of qualitative and quantitative studies. Front Psychol 10: 153, 2019.

10. Burton GJ and Jauniaux E: What is the placenta? Am J Obstet Gynecol 213: S6.e1, S6-8, 2015.

11. Reijnders IF, Mulders AGMGJ and Koster MPH: Placental development and function in women with a history of placenta-related complications: A systematic review. Acta Obstet Gynecol Scand 97: 248-257. 2018.

12. Marlar S, Jensen HH, Login FH and Nejsum LN: Aquaporin-3 in Cancer. Int J Mol Sci 18: pii: E2106, 2017.

13. Frank Wolf M, Peleg D, Stahl-Rosenzweig T, Kurzweil Y and Yogev Y: Isolated polyhydramnios in the third trimester: Is a gestational diabetes evaluation of value? Gynecol Endocrinol 33: 849-852, 2017.

14. Beall MH, Wang S, Yang B, Chaudhri N, Amidi F and Ross MG: Placental and membrane aquaporin water channels: Correlation with amniotic fluid volume and composition. Placenta 28 : 421-428, 2007.

15. Korkmazer E and Solak N: Correlation between inflammatory markers and insulin resistance in pregnancy. $\mathrm{J}$ Obstet Gynaecol 35: 142-145, 2015.

16. Li Z, Li B, Zhang L, Chen L, Sun G, Zhang Q, Wang J, Zhi X, Wang L, Xu Z and Xu H: The proliferation impairment induced by AQP3 deficiency is the result of glycerol uptake and metabolism inhibition in gastric cancer cells. Tumor Biol 37: 9169-9179, 2016.

17. Kawwass JF, Summer R and Kallen CB: Direct effects of leptin and adiponectin on peripheral reproductive tissues: A critical review. Mol Hum Reprod 21: 617-632, 2015.

18. Ott R, Stupin JH, Melchior K, Schellong K, Ziska T, Dudenhausen JW, Henrich W, Rancourt RC and Plagemann A: Alterations of adiponectin gene expression and DNA methylation in adipose tissues and blood cells are associated with gestational diabetes and neonatal outcome. Clin Epigenetics 10: 131, 2018.

19. Chakraborti CK: Role of adiponectin and some other factors linking type 2 diabetes mellitus and obesity. World J Diabetes 6 : $1296-1308,2015$

20. Santana MG, de Velasco PC, Oliveira ORC, Santo RE, Spreafico F, Almeida LB, Sardinha FLC and Tavares-do-Carmo MDG: Adiponectin, insulin and leptin levels in the cord plasma of the neonates from adolescent and adult mothers and their relationship with anthropometric parameters and fetal sex-gender. J Perinatol 38: 489-495, 2018.

21. Li Q, Xiong R, Wang L, Cui J, Shi L, Liu Y and Luo B: Luo, Associations of dietary habits, physical activity and cognitive views with gestational diabetes mellitus among Chinese women. Public Health Nutr 17: 1850-1857, 2014.

22. Feng H, Zhu WW, Yang HX, Wei YM, Wang C, Su RN, Hod M and Hadar E: Relationship between oral glucose tolerance test characteristics and adverse pregnancy outcomes among women with gestational diabetes mellitus. Chin Med J (Engl) 130: 1012-1018, 2017

23. Casey BM, Duryea EL, Abbassi-Ghanavati M, Tudela CM, Shivvers SA, McIntire DD and Leveno KJ: Glyburide in women with mild gestational diabetes. Obstet Gynecol 126: 303-309, 2015.
24. Schmittgen TD and Livak KJ: Analyzing real-time PCR data by the comparative CT method. Nat Protoc 3: 1101-1108, 2008.

25. Correa PJ, Vargas JF, Sen S and lllanes SE: Prediction of gestational diabetes early in pregnancy: Targeting the long-term complications. Gynecol Obstet Inves 77: 145-149, 2014.

26. Lowe LP, Metzger BE, Lowe WL Jr, Dyer AR, McDade TW, McIntyre HD; HAPO Study Cooperative Research Group: Inflammatory mediators and glucose in pregnancy: Results from a subset of the hyperglycemia and adverse pregnancy outcome (HAPO) study. J Clin Endocr Metab 95: 5427-5434, 2010.

27. Fasshauer M, Blüher M and Stumvoll M: Adipokines in gestational diabetes. Lancet Diabetes Endo 2: 488-499, 2014.

28. Lingying $\mathrm{K}$ and Huixia Y: Lipid metabolism and transfer across placenta in women with gestational mellitus. Chin J Obstet Gynecol Pediatr 9: 5-8, 2013.

29. Herrera E and Ortega-Senovilla H: Lipid metabolism during pregnancy and its implications for fetal growth. Curr Pharm Biotechno 15: 24-31, 2014.

30. Gorgal R, Gonçalves E, Barros M, Namora G, Magalhães A, Rodrigues $\mathrm{T}$ and Montenegro N: Gestational diabetes mellitus: A risk factor for non-elective cesarean section. J Obstet Gynaecol Re 38: 154-159, 2012.

31. Zhu X, Jiang S, Hu Y, Zheng X, Zou S, Wang Y and Zhu X: The expression of aquaporin 8 and aquaporin 9 in fetal membranes and placenta in term pregnancies complicated by idiopathic polyhydramnios. Early Hum Dev 86: 657-663, 2010.

32. Jiang SS, Zhu XJ, Ding SD, Wang JJ, Jiang LL, Jiang WX and Zhu XQ: Expression and localization of aquaporins 8 and 9 in term placenta with oligohydramnios. Reprod Sci 19: 1276-1284, 2012.

33. Enquobahrie DA, Williams MA, Qiu C, Meller M and Sorensen TK: Global placental gene expression in gestational diabetes mellitus. Am J Obstet Gynecol 200: 206.e1-206.e13, 2009.

34. Zhi X, Tao J, Li Z, Jiang B, Feng J, Yang L, Xu H and Xu Z: MiR-874 promotes intestinal barrier dysfunction through targeting AQP3 following intestinal ischemic injury. FEBS Lett 588: 757-763, 2014

35. Huang LT, Wu SL, Liao X, Ma SJ and Tan HZ: Adiponectin gene polymorphisms and risk of gestational diabetes mellitus: A meta-analysis. World J Clin Cases 7: 572-584, 2019.

36. Thagaard IN, Hedley PL, Holm JC, Lange T, Larsen T, Krebs L and Christiansen $\mathrm{M}$ : Leptin and adiponectin as markers for preeclampsia in obese pregnant women, a cohort study. Pregnancy Hypertens 15: 78-83, 2019.

37. Altinova AE, Toruner F, Bozkurt N, Bukan N, Karakoc A, Yetkin I, Ayvaz G, Cakir N and Arslan M: Circulating concentrations of adiponectin and tumor necrosis factor- $\alpha$ in gestational diabetes mellitus. Gynecol Endocrinol 23: 161-165, 2007.

38. Benaitreau D, Dos Santos E, Leneveu MC, De Mazancourt P, Pecquery R and Dieudonné MN: Adiponectin promotes syncytialisation of BeWo cell line and primary trophoblast cells. Reprod Biol Endocrinol 8: 128-139, 2010

39. Shen Q, Wang J, Zhou Q, Shen Z, Luo H, Tao X and Zhu X: Linking expression of aquaporin 3 to activation of JNK pathway, Front Biosci 22: 258-267, 2017.

40. Tardelli M, Claudel T, Bruschi FV, Moreno-Viedma V and Trauner M: Adiponectin regulates AQP3 via PPAR $\alpha$ in human hepatic stellate cells. Biochem Bioph Res Commun 490: 51-54, 2017.

This work is licensed under a Creative Commons Attribution-NonCommercial-NoDerivatives 4.0 International (CC BY-NC-ND 4.0) License. 